\title{
BMJ Open Can a sleep disorder intervention- embedded self-management programme contribute to improve management of diabetes? A pilot single-arm pretest and post-test study
}

\author{
Ritsuko Sakamoto, ${ }^{1}$ Kana Kazawa (D) , 2 Yasmin Jahan, ${ }^{1}$ Naoko Takeyama, ${ }^{1}$ \\ Michiko Moriyama $^{1}$
}

To cite: Sakamoto R, Kazawa $\mathrm{K}$, Jahan $\mathrm{Y}$, et al. Can a sleep disorder intervention-embedded self-management programme contribute to improve management of diabetes? A pilot single-arm pretest and post-test study. BMJ Open 2021;11:e045783. doi:10.1136/ bmjopen-2020-045783

- Prepublication history and additional supplemental material for this paper are available online. To view these files, please visit the journal online (http://dx.doi.org/10.1136/ bmjopen-2020-045783).

Received 12 0ctober 2020 Accepted 26 August 2021

Check for updates

(C) Author(s) (or their employer(s)) 2021. Re-use permitted under CC BY-NC. No commercial re-use. See rights and permissions. Published by BMJ.

${ }^{1}$ Chronic Care and Family Nursing, Division of Nursing Science, Graduate School of Biomedical and Health Sciences, Hiroshima University Graduate School, Hiroshima, Japan ${ }^{2}$ Department of Medicine for Integrated Approach to Social Inclusion, Graduate School of Biomedical and Health Sciences, Hiroshima University Graduate School, Hiroshima, Japan

Correspondence to

Dr Kana Kazawa;

kkazawa@hiroshima-u.ac.jp

\section{ABSTRACT}

Objective To investigate the efficacy and feasibility of a self-management programme incorporating a sleep intervention for improving diabetes outcomes.

Design A single-arm pre-test and post-test study was conducted within a community setting in Hiroshima, Japan.

Participants Participants were aged 52-74 years and diagnosed with type 2 diabetic nephropathy stages 1-3. Interventions Participants received self-management education from nurses for 6 months. First, the nurses assessed their sleep conditions using insomnia scales and a sleep metre. Then, the participants learnt selfmanagement to increase their physical activity and improve their sleep condition. They also implemented diet therapy and medication adherence.

Outcome measures Physiological indicators, subjective and objective indicators of sleep quality, self-management indicators, quality of life (QOL) and feasibility were evaluated. To confirm the efficacy of intervention, Freidman tests, analysis of variance, Wilcoxon signed-rank test and t-test were performed. Pearson's correlations were analysed between activities and sleep condition. Results Of the 26 enrolled participants, 24 completed the programme and were analysed. Among them, 15 participants $(62.5 \%)$ had sleep disorders caused by multiple factors, such as an inappropriate lifestyle and physical factors that interfere with good sleep. Although insomnia scales did not change for the sleep disorders, their subjective health status improved. Regarding indicators related to diabetes management, lifestyles improved significantly. Haemoglobin A1c, body mass index, systolic blood pressure, non-high-density lipoproteincholesterol and QOL also improved. All participants except one were satisfied with the programme. However, use of the sleep metre and nurses' consultation about sleep disturbance were not well evaluated.

Conclusions This programme was effective in improving diabetes status, lifestyle and behaviour changes. However, its effect on sleep condition was limited because of its complexity. A simple and novel approach is needed to strengthen the motivation for sleep behaviour change and to increase programme efficacy and feasibility.
Strengths and limitations of this study

- To identify the potential sleep disorders, we used a sleep metre for objective assessment in addition to subjective sleep assessment measures.

- We developed a comprehensive self-management programme incorporating a sleep intervention as sleep disorders have serious consequences for diabetes mellitus management.

- Our limitations relate to statistical power, as this project was a pilot study; a small sample size and a non-controlled study design were implemented.

Trial registration number UMIN000025906.

\section{INTRODUCTION}

The relationship between sleep disorders and impaired glucose tolerance (IGT) is receiving expanded consideration globally due to the negative health outcomes. ${ }^{1-3}$ Previous studies have reported that sleep disorders may disturb glucose homeostasis in complex ways, such as by inducing excessive secretion of stress hormones, which in turn are emphatically related to obesity and type 2 diabetes mellitus (DM) ${ }^{45}$ As DM and IGT are associated with a higher risk of developing cardiovascular disease and death, prevention and management of these health problems are important public health goals. Studies have shown that the prevalence of obstructive sleep apnoea (OSA) in patients with diabetes is estimated to be $23 \%-48 \% .{ }^{67}$ Patients with OSA and type $2 \mathrm{DM}$ are at increased risk of diabetic peripheral neuropathy, ${ }^{8}$ restless legs syndrome ${ }^{9}$ and frequent urination due to hyperglycaemia. ${ }^{10}$ Furthermore, in patients with type 2 DM, the occurrence of OSA is associated with increased oxidative and nitrosative stress as 
well as impaired microvascular regulation. ${ }^{8}$ Hence, it is plausible that OSA complicating type 2 DM could facilitate the development and progression of microvascular complications including diabetic nephropathy.

Epidemiological studies have also found sleep disturbances in patients with diabetes to be correlated with not only glycaemic control but also lower self-care adherence and quality of life (QOL). ${ }^{11}{ }^{12}$ Therefore, we focused on sleep disorders as these are serious problems in patients with diabetes, including diabetic nephropathy and often need to be addressed by non-pharmacological interventions such as sleep management.

Moreover, in DM management, exercise therapy has led to improvement in insulin resistance, advancement of glucose uptake by muscle contraction during exercise, enhancement of lipid abnormalities from the norm and cardiopulmonary function, and improvement of subjective well-being and self-esteem. ${ }^{13}{ }^{14}$ In this context, it can be anticipated that increasing physical activity may improve the sleep quality of patients with diabetes. ${ }^{15} 16$

Regarding interventions for sleep disorders, several studies suggest that significant changes in sleep disorders of chronic primary insomnia are introduced by non-pharmacological approaches. ${ }^{17}$ These approaches include interventions such as sleep hygiene education, relaxation, stress management and cognitive therapy. ${ }^{17} 18$ These interventions function as self-management education for patients with DM and may improve their lifestyle, subjective insomnia evaluation indices and blood glucose levels. ${ }^{19}$ However, few studies consider nonpharmacological treatments in patients with diabetes, including diabetic nephropathy, by screening for sleep disorders using both subjective and objective assessment as well as by analysing the factors that interfere with sleep.

Nonetheless, few DM programmes include most appropriate improvement strategies, depending on the consequences of an assessment of the types and causes of sleep disorders. As yet, there are no reports discussing which of the multiple subjective and objective evaluation tools are most suitable for sleep disorders.

Therefore, this study, as a pilot, aims to evaluate the efficacy of a diabetes self-management programme incorporating sleep disorder assessment and education as well as the feasibility of the programme.

\section{METHODS}

\section{Study design}

This was a pilot, open-label, single-arm, pre- and postdesign study conducted among outpatients with diabetic nephropathy at a community setting in Hiroshima Prefecture, Japan.

\section{Participants}

Participants were aged $\geq 20$ and $<74$ years old and were diagnosed with type $2 \mathrm{DM}$, including diabetic nephropathy.

The inclusion criteria were as follows: (1) diabetic nephropathy stages 1-3 (estimated glomerular filtration rate; eGFR) $\left.>30 \mathrm{~mL} / \mathrm{min} / 1.73 \mathrm{~m}^{2}\right),{ }^{20}$ (2) undergoing treatment for DM on an outpatient basis and (3) insured by National Health Insurance living in Hiroshima City; Union National Health Insurance, the insurer of Japan Health Insurance Association, Hiroshima chapter; and the National Federation of Health Insurance Societies of Hiroshima living in Hiroshima Prefecture.

Patients were excluded if any of the following criteria presents: (1) presence of type 1 or secondary DM; (2) currently hospitalised; (3) undergoing renal replacement therapy or planning to begin renal replacement therapy within the next 6 months; (4) pregnant at the time of the study; (5) in terminal stages; (6) cognitively impaired (score $\leq 20 / 30$ on the revised version of Hasegawa's Dementia Scale ${ }^{21}$; (7) judged by the nurses/primary physicians to be unable to implement any kind of activity required in this study; or (8) already registered in other clinical trials. Patients who were on any kind of sleep medication or psychotropic drugs were not excluded.

As this was an exploratory pilot study, sample size calculation was not performed.

\section{Recruitment and registration}

Participants were introduced to the study by the primary physicians. They were checked for eligibility by the nurses, who obtained informed consent and registered the patients for this study between January and December 2017.

\section{Evaluation of outcomes and data collection schedule}

To evaluate the efficacy and feasibility of the programme incorporating sleep disorder assessment and strengthening exercises, the following parameters were assessed:

- Physiological indicators: body mass index (BMI), systolic blood pressure (SBP), diastolic blood pressure, haemoglobin A1c (HbA1c), eGFR, non-highdensity lipoprotein-cholesterol (non-HDL-c) and triglycerides.

- Subjective measurement of sleep quality using the following: (1) the Insomnia Severity Index, Japanese version (ISI), to evaluate the severity of primary insomnia (cut-off point $\geq 8$ ) ${ }^{22}$; (2) the Pittsburgh Sleep Quality Index, Japanese version (PSQI), to collect information on various domains of sleep including sleep latency, sleep length, sleep efficiency, sleep difficulty, use of sleeping pills, difficulty in waking during the day and secondary insomnia such as OSA (cut-off point $\geq 6)^{23}$; and (3) the Japanese version of Epworth Sleepiness Scale (JESS), to evaluate the excessive daytime sleepiness (cut-off point $\geq 11$ ). ${ }^{24}$

- Objective measurement of sleep quality: a sensor mattype sleep metre (NEMURI SCAN NN-1310; Paramount bed Co) was used to distinguish between sleep and wakefulness. Although the measured activity is different between whole body movements and wrist movements, this actigraphy device can measure sleep/wake states with almost the same accuracy as wrist actigraphy. ${ }^{25}$ Moreover, this actigraphy device 


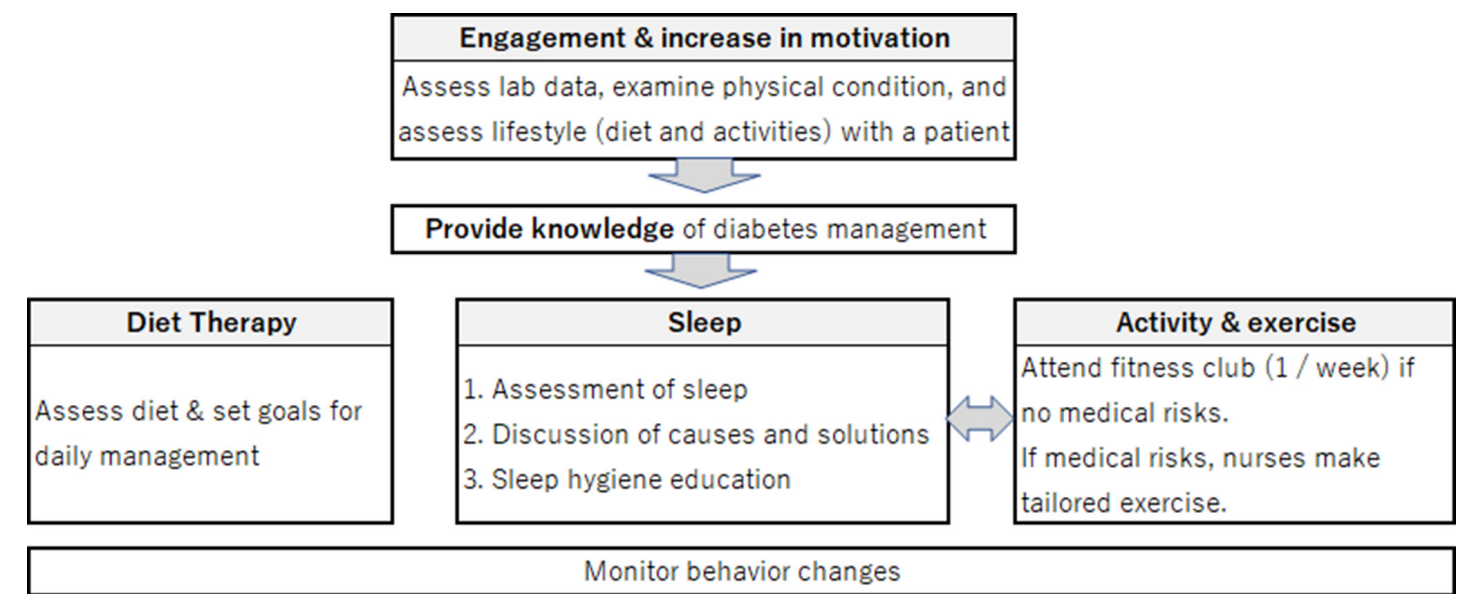

Figure 1 Framework of the intervention programme.

can be placed under the bed or mattress and can be easily used at home. This device records total sleep time, time in bed, sleep latency, sleep efficiency, wakefulness after sleep onset, number of out-of-bed instances, respiratory event index, periodical body movement index, activity score, respiratory rate, heart rate, sleep/wake log (graph showing sleep and wake patterns are colour coded). It then evaluates sleep on a scale from 1 to 4 (good), where 3 implies 'no abnormality', 2 implies 'attention required', and 1 implies 'improvement required'. A score of 2 or less implies a possible sleep disorder (online supplemental file 1).

- Self-management behaviour: the nurses asked the participants for the frequency of blood pressure (BP) and body weight self-monitoring, diet (prioritising vegetable consumption, etc) and activity/exercise. The participant's activity/exercise level was measured as the total number of steps per day using a pedometer (Lifecorder GF, Suzuken Co, Japan).

- Health-related QOL: EuroQOL-5D-5L, Japanese version, was administered to evaluate the overall outcome of this programme. ${ }^{27}$ This questionnaire evaluates five items (mobility, self-care, usual activities, pain/discomfort and anxiety/depression), with each item evaluated on a scale of 1-5 (no problems, slight problems, moderate problems, severe problems and extreme problems). For the subjective health status included in the EuroQOL tool, the participants make a self-assessment of their health on a scale from 0 (worst condition) to 100 (best condition). The EuroQOL-5D-5L was scored using tariffs.

During the 6-month intervention period, physiological and self-management indicators were collected every 3 months, while data regarding other variables were collected at baseline and at 6 months.

\section{Definition of sleep disorder}

Sleep disorders include seven major categories in the International Classification of Sleep Disorders-Third Edition: insomnia, sleep-related breathing disorders, central disorders of hypersomnolence, circadian rhythm sleep-wake disorders, sleep-related movement disorders, parasomnias and other sleep disorders. ${ }^{28}$ In this study, we defined patients with sleep disorders as those having been diagnosed with a sleep disorder at a specialist clinic and/ or those having awareness of their insomnia for more than 1 month and meeting at least one criteria (ISI $\geq 8$ points, $\mathrm{PSQI} \geq 6$ points, JESS $>11$ points) on the sleep questionnaire or via the sleep metre's comprehensive judgement.

\section{Self-management intervention programme incorporating sleep improvement and activity/exercise enhancement}

Figure 1 describes the framework of the intervention programme in this study. The programme was originally designed for patients to acquire skills for self-management of diabetic nephropathy. ${ }^{29}$ The programme was implemented for 6 months and monitored via face-to-face and telephonic interviews.

After patient enrolment, the nurses performed a comprehensive assessment of laboratory test results; physical conditions; lifestyle practices such as diet, activities/ exercise, drug adherence, alcohol, smoking, and psychosocial status of the participants; they also discussed the aggravating factors of DM. The nurses also explained the stage of diabetic nephropathy, treatment goals of the stage and self-management using educational textbooks created by the authors of this study. The nurses and the participants jointly set the monthly goals and action plans for behavioural changes. The nurses educated the participants on various self-monitoring methods (measurement and evaluation of $\mathrm{BP}$ and body weight). ${ }^{30}$

With regard to increasing physical activity, the nurses introduced the participants to a fitness club if the participant did not have medical risks based on initial assessment by a primary physician. If a participant had medical risks for attending a fitness club or refused to attend, the nurses prescribed tailored exercises based on their physical tolerance and preference. For all participants, the nurses lent a pedometer and asked them to account for their activity for 1 week on baseline measurements. Visits to the fitness club were scheduled once a week during the first 3 months, with attendance 


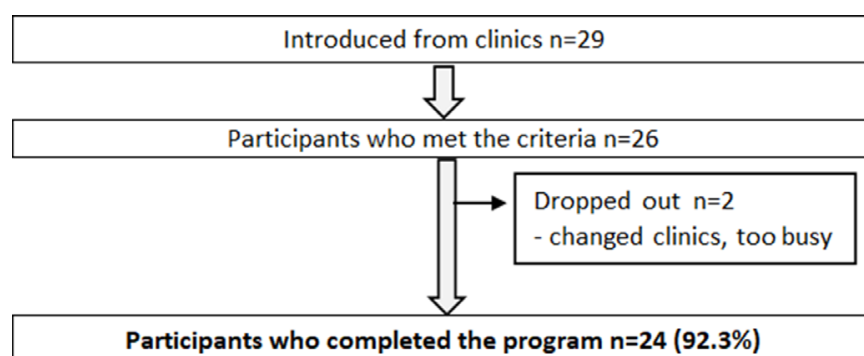

Figure 2 Flow diagram of the participants.

being voluntary after this period. At the fitness club, a health fitness trainer held classes, approximately $60 \mathrm{~min}$ in duration, which involved walking, stretching, squatting and gymnastics (exercise intensity was about 3-5 metabolic equivalents).

Screening, evaluation and intervention for sleep disorders: three subjective assessment scales (ISI, PSQI and JESS) were administered for screening and evaluating the participants' sleeping states. For those who were evaluated as having a sleep disorder on these scales and/ or those who had a BMI of 30 or more (high chance to have sleep apnoea syndrome (SAS)), a sleep metre was lent to them by the nurses with usage instructions, and they were asked to use the device for 1 week. After the sleep metre was returned, the conditions of the participant's sleep disorders based on the subjective evaluation scale and the results of the sleep metre were explained to them by the nurses. The nurses used a researcherdeveloped textbook in which the pathology, factors causing sleep disorders and methods used to both deal with the problem as well as promote sleep hygiene were explained (online supplemental file 2). This textbook was developed based on a qualitative study. ${ }^{31}$ The participants and the nurses then discussed how to solve the problem. The nurses contacted a specialist to evaluate those with moderate or higher levels of sleep disorders or suspected SAS. Those who were subjectively evaluated as not having any sleep disorders were also provided with prophylactic general sleep hygiene education using the textbook.

After the first session, the participants implemented the action plan and recorded their self-monitoring values in a notebook. The nurses evaluated the implementation of the action plan through face-to-face consultations (once every 2nd and 3rd month) and biweekly phone calls, proposing revisions to the implemented action plan when necessary.

\section{Data analysis}

SPSS software V.26.0 was used for analysis. Normality was confirmed for each item, descriptive statistics were calculated, and Freidman tests, analysis of variance, Wilcoxon signed-rank test and t-tests were performed (where appropriate) before and after the intervention. Pearson's correlations between activities and sleep condition were analysed. The significance level was set at $5 \%$.

\section{Patient and public involvement}

The educational textbook was developed based on patients' priorities and patients' experience by conducting qualitative research ${ }^{31}$. As this is a selfmanagement programme, patients were asked to report their data and progress to the researchers. Further, patients evaluated the feasibility of the programme. However, they were not directly involved in the design and conception of the study.

\section{RESULTS}

\section{Baseline characteristics of the participants}

Twenty-nine participants were introduced by five primary physicians, among them 26 met the eligibility criteria and were enrolled. Of the 26 patients, 17 were male $(65.4 \%)$, and the mean age was $65.7 \pm 6.0$ years. Two participants dropped out right after enrolment. Therefore, only 24 participants completed the 6-month programme and were included in the analysis (figure 2). Of the 24 participants, 15 (62.5\%) were classified as having sleep disorders. Eleven participants $(45.8 \%)$ had sleep disorders based on any of the sleep questionnaires (ISI, PSQI, JESS). Eight had an ISI score of 8 or greater, nine had a PSQI score of 6 or greater, and two had a JESS score of 11 or greater. Moreover, 13 out of the $15(86.7 \%)$ participants who agreed to use a sleep metre had sleep disorders. However, four of them were not screened as having sleep disorders based on the sleep questionnaire (ie, those who were not aware that their sleep quality was poor indeed) (table 1). In contrast, two participants were screened as having sleep disorders on the sleep questionnaire but assessed as 'no abnormality' on the sleep metre. Of the three questionnaires, PSQI could identify the most sleep disorders and the identified sleep disorders that could not be extracted by other scales.

According to the nurses' comprehensive assessment of 15 participants, factors which disturbed sleep included inappropriate lifestyle behaviours such as intake of alcohol before going to bed, lack of activity during the daytime, and using a computer or smartphone before going to bed. We also found that most participants have several other physical conditions, like polyuria and pain. Suspected SAS and periodical limb movement disorders were also found in six and three participants, respectively.

To understand the characteristics of participants with sleep disorders, we compared their baseline data with those without sleep disorders (table 2). Although the divided sample size was small and difficult to compare, the results showed that participants with sleep disorders had higher BMI, BP and lipid profile, and lower renal function and subjective health status at baseline.

\section{Efficacy of the programme (one-way analysis)}

To examine programme efficacy, baseline conditions were compared with outcomes during the 3rd and 6th months of intervention (table 3, figure 3). BMI, SBP, 
Table 1 Assessment results of the participants' sleep at baseline $n=24$

\begin{tabular}{|c|c|c|c|c|c|c|}
\hline \multirow{2}{*}{$\begin{array}{l}\text { Number of people with } \\
\text { sleep disorders } \mathrm{n}(\%)\end{array}$} & \multicolumn{4}{|c|}{ Applicable to sleep disorder on other scales } & \multirow{2}{*}{$\begin{array}{l}\text { Not applicable to sleep } \\
\text { disorders on any other scales }\end{array}$} & \multirow[b]{2}{*}{ Total number } \\
\hline & ISI & PSQI & JESS & Sleep metre & & \\
\hline ISI ( $\leq 8$ points) & & $6(75.0)$ & $2(25.0)$ & $8(100.0)$ & $0(0.0)$ & $8(100.0)$ \\
\hline JESS ( $\leq 11$ points) & $2(100.0)$ & $1(50.0)$ & & $2(100.0)$ & $0(0.0)$ & $2(100.0)$ \\
\hline Sleep metre ( $\leq 2$ points) & $8(61.5)$ & 7 (53.8) & $2(15.4)$ & & $4(30.8)$ & $13(100.0)$ \\
\hline
\end{tabular}

Of the 26 who agreed to participate in the study, 2 could not be evaluated for sleep because they declined immediately after consent.

ISI, Insomnia Severity Index; JESS, Japanese version of Epworth Sleepiness Scale; PSQI, Pittsburgh Sleep Quality Index.

non-HDL-c and HbA1c improved significantly $(\mathrm{p}<0.05)$. Renal functions (eGFR) did not change. QOL score and subjective health status also improved significantly $(\mathrm{p}<0.05)$. Considering the sleep questionnaires, there was no significant difference between the scores before and after the intervention (median $(\mathrm{IQR})=1 \quad(1-2)$, 2 (1-2.25), respectively, $\mathrm{p}=0.317, \mathrm{Z}=-1.000)$. Activity levels measured by steps were maintained but did not show any significant improvement $(p=0.271)$. Changes in all self-management behaviours improved significantly $(\mathrm{p}<0.05)$.

One participant started while another was being tapered off antihypertensive medication during the study. There was no change in the prescription for hypoglycaemic drugs. One participant was being tapered off insulin. However, no participant had started taking sleep medication during the study.
To examine how the increase in exercise improved sleep status, we analysed the relationship between them at the end of the 6-month programme for all 24 participants (figure 4). The results showed that the ISI score and exercise level (number of steps per day) were moderately negatively correlated $(\mathrm{r}=0.395, \mathrm{p}=0.056)$. In other words, the greater the amount of exercise, the lower the severity of insomnia and the better the quality of sleep. There were positive relationships among the PSQI (degree of sleep disorder) score, JESS (sleepiness in the daytime) score, scores on the sleep metre and exercise frequency, but these were not statistically significant.

\section{Feasibility of the programme}

All participants except one $(96 \%)$ were satisfied with this programme, the nurses' intervention and the fitness club for those who attended it. In contrast, the use of the sleep

Table 2 Comparison of baseline feature in groups with and without sleep disorders

\begin{tabular}{|c|c|c|c|c|}
\hline & \multicolumn{4}{|c|}{ Mean $\pm S D$} \\
\hline & \multicolumn{2}{|c|}{ Sleep disorders $(n=15)$} & \multicolumn{2}{|c|}{ Without sleep disorders $(n=9)$} \\
\hline & $\mathbf{n}$ & & $\mathbf{n}$ & \\
\hline \multicolumn{5}{|l|}{ Physiological indicators } \\
\hline BMI & 15 & $26.3 \pm 6.2$ & 9 & $23.7 \pm 2.3$ \\
\hline eGFR (mL/min/1.73 m²) & 15 & $64.5 \pm 22.5$ & 9 & $67.6 \pm 9.3$ \\
\hline Triglyceride (mg/dL ) & 14 & $191.6 \pm 121.7$ & 9 & $148.3 \pm 88.7$ \\
\hline non-HDL-c (mg/dL ) & 14 & $146.9 \pm 41.1$ & 9 & $126 \pm 19.6$ \\
\hline $\mathrm{HbA1c}(\%)$ & 15 & $8.0 \pm 1.0$ & 9 & $8.0 \pm 1.4$ \\
\hline Number of steps per day & 11 & $6819.7 \pm 2399$ & 9 & $8915.3 \pm 3022.5$ \\
\hline \multicolumn{5}{|l|}{ Quality of sleep } \\
\hline ISI (score) & 15 & $8.4 \pm 3.8$ & 9 & $3.1 \pm 2.2$ \\
\hline PSQI (score) & 15 & $6.2 \pm 2.2$ & 9 & $2.7 \pm 1.9$ \\
\hline JESS (score) & 15 & $5.2 \pm 3.5$ & 8 & $3.8 \pm 2.2$ \\
\hline
\end{tabular}

BMI, body mass index; DBP, diastolic blood pressure; eGFR, estimated glomerular filtration rate; HbA1c, haemoglobin A1c; HDL-c, high-density lipoprotein-cholesterol; ISI, Insomnia Severity Index; JESS, Japanese version of Epworth Sleepiness Scale; PSQI, Pittsburgh Sleep Quality Index; QOL, quality of life; SBP, systolic blood pressure. 
Table 3 Changes in outcomes

\begin{tabular}{|c|c|c|c|c|c|}
\hline & \multicolumn{5}{|c|}{ Mean \pm SD } \\
\hline & $n$ & Baseline & 3rd month & 6th month & $P$ value \\
\hline \multicolumn{6}{|l|}{ Physiological indicators } \\
\hline BMI & 24 & $25.3 \pm 5.2$ & $24.7 \pm 5.2$ & $24.2 \pm 5.1$ & $<0.001^{*}$ \\
\hline SBP $(\mathrm{mm} \mathrm{Hg})$ & 20 & $138.4 \pm 14.1$ & $129.3 \pm 15.5$ & $125.8 \pm 15.4$ & $0.009 \dagger$ \\
\hline $\mathrm{DBP}(\mathrm{mm} \mathrm{Hg})$ & 20 & $75.8 \pm 11.8$ & $70.4 \pm 8.1$ & $73.2 \pm 11.9$ & $0.156 \dagger$ \\
\hline eGFR $\left(\mathrm{mL} / \mathrm{min} / 1.73 \mathrm{~m}^{2}\right)$ & 24 & $65.7 \pm 18.4$ & - & $63.0 \pm 13.1$ & $0.886 \ddagger$ \\
\hline Triglyceride (mg/dL ) & 23 & $174.0 \pm 110$ & - & $165.2 \pm 78.5$ & $0.976 \ddagger$ \\
\hline non-HDL-c (mg/dL ) & 23 & $138.8 \pm 35.3$ & - & $122.3 \pm 30.5$ & $0.017 \S$ \\
\hline $\mathrm{HbA1c}(\%)$ & 24 & $8.0 \pm 1.2$ & $7.4 \pm 1.1$ & $7.0 \pm 0.9$ & $<0.001^{*}$ \\
\hline Number of steps per day & 20 & $7762.8 \pm 2831.9$ & $7597.4 \pm 3509.1$ & $7247.8 \pm 3331.5$ & $0.271 \dagger$ \\
\hline \multicolumn{6}{|l|}{ QOL } \\
\hline EuroQOL-5D-5L (score) & 24 & $0.86 \pm 0.11$ & - & $0.89 \pm 0.12$ & $0.031 \S$ \\
\hline Subjective health status (\%) & 24 & $75.1 \pm 16.8$ & - & $80.9 \pm 14.5$ & $0.016 \S$ \\
\hline \multicolumn{6}{|l|}{ Quality of sleep } \\
\hline ISI (score) & 24 & $6.4 \pm 4.2$ & - & $6.4 \pm 4.8$ & $1.000 \S$ \\
\hline PSQI (score) & 24 & $4.9 \pm 2.7$ & - & $5.5 \pm 3.4$ & $0.207 \S$ \\
\hline JESS (score) & 23 & $4.7 \pm 3.2$ & - & $4.5 \pm 3.2$ & $0.739 \S$ \\
\hline
\end{tabular}

${ }^{\star}$ Friedman test.

†ANOVA.

¥Wilcoxon signed-rank test.

$\S$ T-test.

ANOVA, analysis of variance; BMI, body mass index; DBP, diastolic blood pressure; eGFR, estimated glomerular filtration rate; HbA1c, haemoglobin A1c; HDL-c, high-density lipoprotein-cholesterol; ISI, Insomnia Severity Index; JESS, Japanese version of Epworth Sleepiness Scale; PSQI, Pittsburgh Sleep Quality Index; QOL, quality of life; SBP, systolic blood pressure.

metre and the consultation that the nurse gave regarding sleep disorders were negatively evaluated (they were evaluated as 'useful' by $36 \%$ and $30 \%$, respectively).

Attending a fitness club was highly evaluated based on the free comments, as the participants acquired exercise habits and had fun learning the correct techniques of doing the exercises. Participants who underwent sleep assessment and consultation were more likely to acknowledge the importance of awareness and understanding of their sleep conditions and how they were related to DM. For instance, one participant reported that his snoring disappeared. In contrast, most participants tended to make negative comments stating that the use of the sleep metre was bothersome and difficult to implement, and that they were likely to discontinue its use. Some participants were frustrated and refused to change their behaviours, saying "even if you point out the sleep problem, there is no need to change now, because there is no discomfort."

\section{DISCUSSION}

In this pilot study, we aimed to evaluate the efficacy of the self-management programme for diabetes by incorporating measures aimed at improving sleep and increasing physical activity. Moreover, to detect the persons who had sleep disorders, we applied three subjective questionnaires and an objective sleep metre for sleep assessment.

We found that $62.5 \%$ of the participants had sleep disorders, which was higher than the previously reported studies involving patients with diabetes. ${ }^{32}{ }^{33}$ It is important to note that our study is different from these previous studies, since these evaluated sleep disorders using subjective surveys. However, in this study, sleep disorders were objectively evaluated as well. For instance, a few participants were not aware that they had insomnia, which was determined only through objective evaluation using a sleep metre. A previous study reported that patients with diabetes may have difficulty in perceiving sleep disorders due to neuropathy. ${ }^{34}$ Therefore, our results suggest that it is useful to identify high-risk individuals with sleep disorders and recommend that a sleep metre needs to be used first before administering the questionnaires. When individuals refuse to use a sleep metre, the PSQI, which broadly identifies insomnia, should be administered. As a majority of the participants in this study negatively evaluated the use of sleep metres, medical professionals will need to emphasise the importance of objective assessment.

In terms of preventing aggravation of the early stage of diabetic nephropathy, the physiological and behavioural results of this study suggest that this integrated programme 


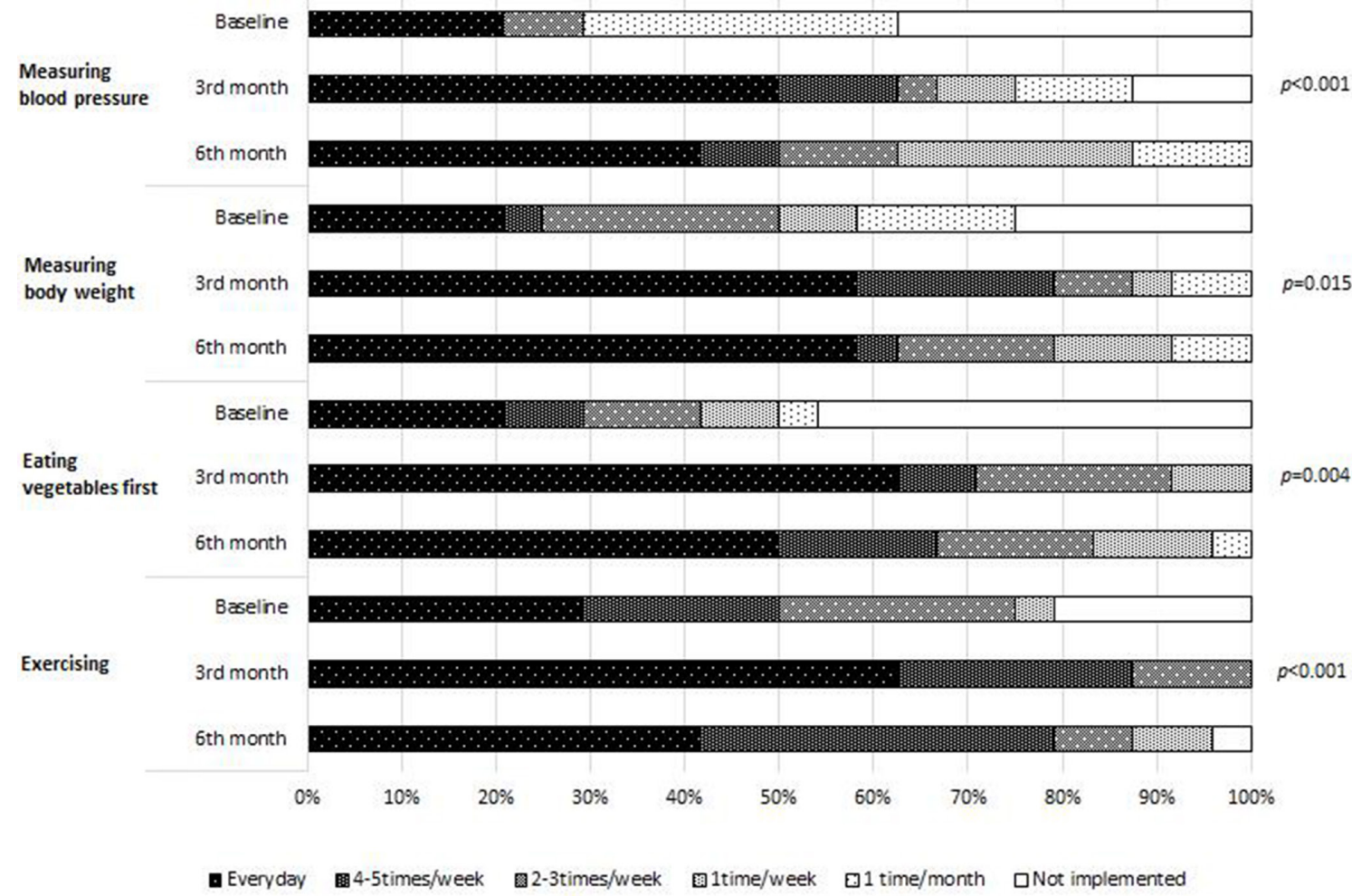

Note: Friedman test

Figure 3 Changes in self-management behaviours. Note: Friedman test.

was effective, except for use in sleep interventions due to the small sample size. Our pilot study found that all the participants showed significant improvements in self-management activities by eating healthy meals, exercising and self-monitoring. This resulted in a significant improvement in BMI, SBP and HbAlc, which further decreases the future development of vascular complications. ${ }^{35}$ Subsequently, patients' QOL scores and subjective assessment of health improved significantly. However, few patients with type $2 \mathrm{DM}$ felt the disease-specific distress to maintain self-management of DM throughout their lives. ${ }^{36} 37$ The structured behavioural self-management education evaluated in this study might have improved the participants' subjective rating of health. ${ }^{37}$

Regarding the relationship between exercise frequency and changes in sleep state, ISI scores decreased (improved) and sleep metre score increased (improved), as exercise frequency increased. This result indicated that, as expected, increasing exercise frequency contributed to improvements in sleep condition, although statistically significant improvements were not observed in the quality of sleep itself. Many studies have reported the positive relationship between exercise and quality of sleep by improving the symptoms seen in respiratory-related sleep disorders. Moreover, continuous exercise and weight loss were found to improve glycaemic control. ${ }^{1338}$ These positive effects might have been affected by peer support and group dynamics based on the comments of participants who used the fitness club. ${ }^{39}$ Therefore, in a diabetes self-education programme incorporating sleep improvement, performing periodical exercise by enrolling in fitness clubs may be beneficial.

In this study, the participants' evaluation of sleep assessment and nurses' consultation for sleep improvement were poorly evaluated, and the results did not support sleep improvement. The participants in this study had poor glycaemic control compared with patients with type $2 \mathrm{DM}$ with sleep disorders which is consistent with other interventional studies. ${ }^{19} 40$ Additionally, although there was a high prevalence of sleep disorders in this population and multiple sleep-inhibitory factors such as suspicion of SAS, pain and unhealthy lifestyles were present in patients with sleep disorders, most participants did not recognise that they had sleep disorders or that it could aggravate DM. Therefore, there was a need for sleep assessment, motivating tailored education and referral to a specialist. Since DM with sleep disorders have several risks, this programme needs to be implemented for a longer period of time, with the content taught in the following order-DM education first, followed by sleep education/problem-solving, which will be more realistic and acceptable.

\section{Limitations}

As this project was a pilot study, a small sample size and a non-controlled study design were implemented, which made it difficult to analyse with strong statistical power. Besides, due to the small sample size, we cannot compare the sleep intervention effect itself as the physical 
ISI score

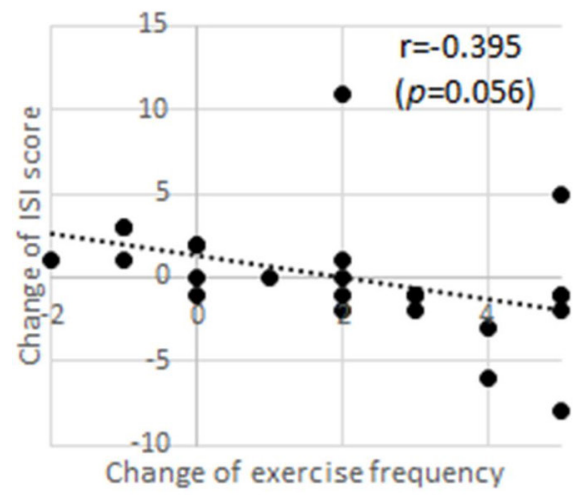

ESS score

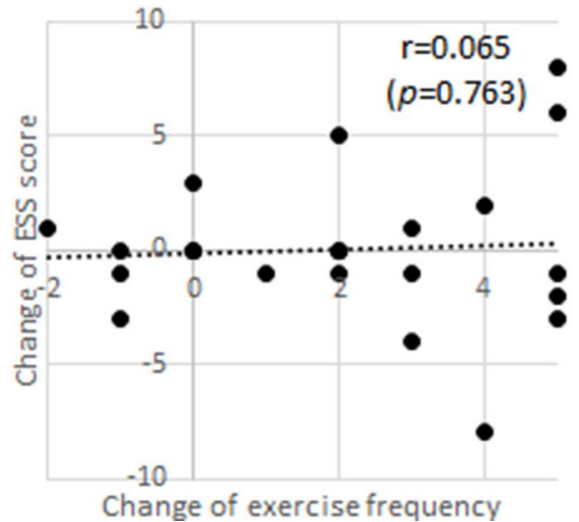

PSQI score

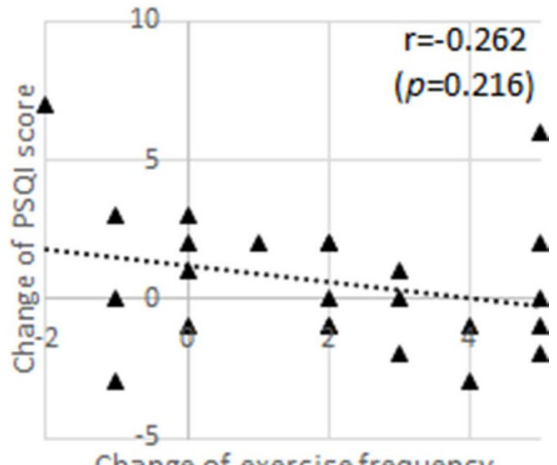

Change of exercise frequency

Sleep meter score

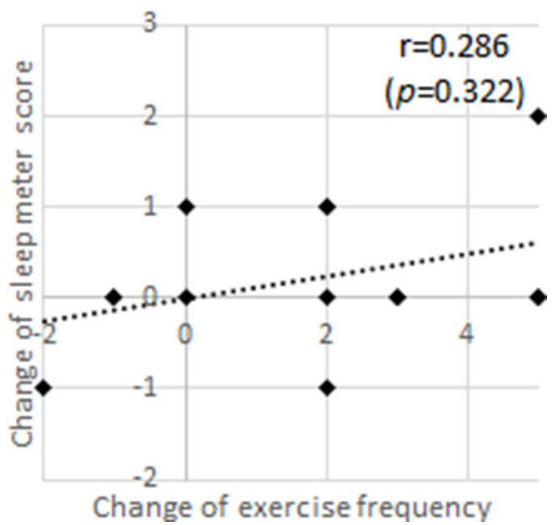

Figure 4 Relationship between the sleep status and exercise frequency. ESS, Epworth Sleepiness Scale; ISI, Insomnia Severity Index; PSQI, Pittsburgh Sleep Quality Index.

indicators and QOL of participants as a group improved significantly. Additionally, this was a community-based study (local government project) with a complex protocol, which led to missing out certain existing data, making the statistical comparison difficult. In the future, it is necessary to simplify and verify the programme's effect on a larger population as well as account for the prevalence of sleep disorders in the analysis. Moreover, this study had an intervention period of 6 months; hence, it is recommended to establish a longer term intervention and monitoring period because the unpredictability of the factors can hinder sleep in patients with diabetes to completely identify the impacts of this programme. Also, further research with a large sample size should be conducted to evaluate the effectiveness of the intervention.

\section{CONCLUSION}

We conducted a self-management programme for the patients with diabetic nephropathy (up to the third stage) aimed at improving sleep and increasing physical activity. BMI, SBP, HbAlc, QOL scores and subjective health perceptions of all patients improved significantly after the intervention. As for sleep-related evaluation indicators, there were some participants who showed improvement on objective evaluation by the sleep metre but remained unchanged when the subjective evaluation index was used. While the direct impacts of sleep intervention were not observed in these results, the necessity of this programme was highlighted, allowing us to consider approaches to improve it for its full-scale implementation in the future.

Acknowledgements We thank the Hiroshima Prefecture, Division of Integrated Care \& Elderly support and the Hiroshima City, Division of Health \& Welfare, Insurance and Pension Section for initiating and funding this project. We also thank the study participants and their primary physicians for their help in completing this project. We acknowledge the contribution of Madoka Kawai, Muzuho Yamaguchi and Mie Fukushima, who implemented the programme. Our sincere thanks to Naoko Matsumoto, her staff and Health Fitness trainer from Medical Fitness B-1, Hiroshima, for the provision of the study's fitness interventions.

Contributors RS was involved in research design, data collection, analysis and manuscript writing. KK was involved in data analysis and manuscript writing. YJ was invoved in manuscript writing. NT was involved in programme and educational material development. MM was involved in overall research design including programme development and manuscript writing. All authors read and approved the final manuscript.

Funding This work was supported by a Grant-in-Aid for Scientific Research (B) (No. 15H05078) and was funded by Hiroshima Prefecture and Hiroshima City as a joint research.

Competing interests None declared.

Patient consent for publication Obtained.

Ethics approval The study protocol was approved by Hiroshima University Ethics Committee (No. C-140). Written informed consent was obtained from each participant. This study was conducted under the health insurance system of Japan 
and performed in accordance with the Declaration of Helsinki and the Ethical Guidelines on Clinical Studies of the Ministry of Health, Labour and Welfare of Japan.

\section{Provenance and peer review Not commissioned; externally peer reviewed.}

Data availability statement No data are available. This was the joint project of Hiroshima Prefecture and Hiroshima City, and the data cannot be publicly available in order to guarantee the anonymity of participants. No additional data available.

Supplemental material This content has been supplied by the author(s). It has not been vetted by BMJ Publishing Group Limited (BMJ) and may not have been peer-reviewed. Any opinions or recommendations discussed are solely those of the author(s) and are not endorsed by BMJ. BMJ disclaims all liability and responsibility arising from any reliance placed on the content. Where the content includes any translated material, BMJ does not warrant the accuracy and reliability of the translations (including but not limited to local regulations, clinical guidelines, terminology, drug names and drug dosages), and is not responsible for any error and/or omissions arising from translation and adaptation or otherwise.

Open access This is an open access article distributed in accordance with the Creative Commons Attribution Non Commercial (CC BY-NC 4.0) license, which permits others to distribute, remix, adapt, build upon this work non-commercially, and license their derivative works on different terms, provided the original work is properly cited, appropriate credit is given, any changes made indicated, and the use is non-commercial. See: http://creativecommons.org/licenses/by-nc/4.0/.

\section{ORCID iD}

Kana Kazawa http://orcid.org/0000-0002-5303-8542

\section{REFERENCES}

1 Gottlieb DJ, Punjabi NM, Newman AB, et al. Association of sleep time with diabetes mellitus and impaired glucose tolerance. Arch Intern Med 2005;165:863-7.

2 Cappuccio FP, D'Elia L, Strazzullo P, et al. Quantity and quality of sleep and incidence of type 2 diabetes: a systematic review and meta-analysis. Diabetes Care 2010;33:414-20.

3 Zizi F, Jean-Louis G, Brown CD, et al. Sleep duration and the risk of diabetes mellitus: epidemiologic evidence and pathophysiologic insights. Curr Diab Rep 2010;10:43-7.

4 Spiegel K, Knutson K, Leproult R, et al. Sleep loss: a novel risk factor for insulin resistance and type 2 diabetes. J Appl Physiol 2005;99:2008-19.

5 Rosique-Esteban N, Papandreou C, Romaguera D, et al. Crosssectional associations of objectively-measured sleep characteristics with obesity and type 2 diabetes in the PREDIMED-Plus trial. Sleep 2018;41. doi:10.1093/sleep/zsy190. [Epub ahead of print: 0112 2018].

6 Inkster B, Riha RL, Van Look L, et al. Association between excessive daytime sleepiness and severe hypoglycemia in people with type 2 diabetes: the Edinburgh type 2 diabetes study. Diabetes Care 2013;36:4157-9.

7 Bruyneel M, Kleynen P, Poppe K. Prevalence of undiagnosed glucose intolerance and type 2 diabetes in patients with moderate-to-severe obstructive sleep apnea syndrome. Sleep Breath 2020;24:1389-95

8 Tahrani AA, Ali A, Raymond NT, et al. Obstructive sleep apnea and diabetic neuropathy: a novel association in patients with type 2 diabetes. Am J Respir Crit Care Med 2012;186:434-41.

9 Martínez Cerón E, Casitas Mateos R, García-Río F. Sleep apneahypopnea syndrome and type 2 diabetes. A reciprocal relationship? Arch Bronconeumol 2015;51:128-39.

10 Modarresnia L, Golgiri F, Madani NH, et al. Restless legs syndrome in Iranian people with type 2 diabetes mellitus: the role in quality of life and quality of sleep. J Clin Sleep Med 2018;14:223-8.

11 Chasens ER, Korytkowski M, Sereika SM, et al. Effect of poor sleep quality and excessive daytime sleepiness on factors associated with diabetes self-management. Diabetes Educ 2013;39:74-82.

12 Oda A, Inagaki M, Tasaki K, et al. The concept of sleep ability and its effect on diabetes control in adults with type 2 diabetes. Can $J$ Diabetes 2019;43:329-35.

13 Hayes C, Kriska A. Role of physical activity in diabetes management and prevention. J Am Diet Assoc 2008;108:S19-23.

14 Zanuso S, Balducci S, Jimenez A. Physical activity, a key factor to quality of life in type 2 diabetic patients. Diabetes Metab Res Rev 2009;25 Suppl 1:S24-8.

15 Waryasz GR, McDermott AY. Exercise prescription and the patient with type 2 diabetes: a clinical approach to optimizing patient outcomes. J Am Acad Nurse Pract 2010;22:217-27.
16 Balducci S, Sacchetti M, Haxhi J, et al. Physical exercise as therapy for type 2 diabetes mellitus. Diabetes Metab Res Rev 2014;30 Suppl $1: 13-23$.

17 Sivertsen B, Omvik S, Pallesen S, et al. Cognitive behavioral therapy vs zopiclone for treatment of chronic primary insomnia in older adults: a randomized controlled trial. JAMA 2006;295:2851-8.

18 Wickboldt AT, Bowen AF, Kaye AJ, et al. Sleep physiology, abnormal states, and therapeutic interventions. Ochsner J 2012;12:122-34.

19 Khosravan S, Alami A, Golchin Rahni S. Effects of continuous care model based non-pharmacological intervention on sleep quality in patients with type 2 diabetes mellitus: a randomized controlled clinical trial. Int J Community Based Nurs Midwifery 2015;3:96-104

20 The Japan Diabetes Society. Chronic complications. 2 diabetic nephropathy. In: Treatment guide for diabetes, 2020.

21 Kato S, Shimogaki H, Onodera A. Development of the revised version of hasegawa's dementia scale (HDS-R). Japanese $j$ geriatric psychiatry 1991;2:1339-47 https://www.scienceopen.com/ document?vid=1eabfb9b-3b26-47a6-9ba0-eff8f7b16364

22 Munezawa T, Morin CM, Inoue Y. Development of the Japanese version of the insomnia severity index (ISI-J). Japan. Journal of Psychiatry Treatment 2009;24:219-25.

23 Doi Y, Minowa M, Uchiyama M, et al. Psychometric assessment of subjective sleep quality using the Japanese version of the Pittsburgh sleep quality index (PSQI-J) in psychiatric disordered and control subjects. Psychiatry Res 2000;97:165-72.

24 Takegami M, Suzukamo Y, Wakita T, et al. Development of a Japanese version of the Epworth Sleepiness scale (JESS) based on item response theory. Sleep Med 2009;10:556-65.

25 Kogure T, Shirakawa S, Shimokawa M, et al. Automatic sleep/wake scoring from body motion in bed: validation of a newly developed sensor placed under a mattress. J Physiol Anthropol 2011;30:103-9. s.

26 Kogure T, Ebata T. Activity during sleep measured by a SheetShaped body Vibrometer and the severity of atopic dermatitis in adults: a comparison with wrist actigraphy. J Clin Sleep Med 2018;14:199-204.

27 Ikeda S, Shiraiwa T, Igarashi A. Developing a Japanese version of the EQ-5D-5L value set. Journal of the National Institute of Public Health 2015;64:47-55.

28 Sateia MJ. International classification of sleep disorders-third edition: highlights and modifications. Chest 2014;146:1387-94.

29 Kazawa K, Takeshita Y, Yorioka N, et al. Efficacy of a disease management program focused on acquisition of self-management skills in pre-dialysis patients with diabetic nephropathy: 24 months follow-up. J Nephrol 2015;28:329-38.

30 Kazawa K, Moriyama M. Effects of a self-management skillsacquisition program on pre-dialysis patients with diabetic nephropathy. Nephrol Nurs J 2013;40:141-8.

31 Toyoshima A, Moriyama M, Yamashita $\mathrm{H}$, et al. Understanding the process for developing sleep disorders among Japanese workers: a qualitative study. Health Promotion Perspectives 2021;11:87-96.

32 Arosemena Coronel M, Sánchez Armijos J, Tettamanti Miranda $\mathrm{D}$, et al. Excessive daytime somnolence is associated with hypoglycemia in adult Latinos with type 2 diabetes mellitus. Sleep Med 2017;36:6-9.

33 Otaka H, Murakami H, Nakayama H, et al. Association between insomnia and personality traits among Japanese patients with type 2 diabetes mellitus. J Diabetes Investig 2019;10:484-90.

34 Nakajima H, Kaneita Y, Yokoyama E, et al. Association between sleep duration and hemoglobin A1c level. Sleep Med 2008;9:745-52.

35 Gaede $\mathrm{P}$, Lund-Andersen $\mathrm{H}$, Parving $\mathrm{H}-\mathrm{H}$, et al. Effect of a multifactorial intervention on mortality in type 2 diabetes. $N$ Engl J Med 2008;358:580-91.

36 Perrin NE, Davies MJ, Robertson N, et al. The prevalence of diabetes-specific emotional distress in people with type 2 diabetes: a systematic review and meta-analysis. Diabet Med 2017;34:1508-20.

37 Perrin N, Bodicoat DH, Davies MJ, et al. Effectiveness of psychoeducational interventions for the treatment of diabetesspecific emotional distress and glycaemic control in people with type 2 diabetes: a systematic review and meta-analysis. Prim Care Diabetes 2019;13:556-67.

38 Isobe Y, Nakatsumi Y, Sugiyama Y, et al. Severity indices for obstructive sleep apnea syndrome reflecting glycemic control or insulin resistance. Intern Med 2019;58:3227-34.

39 Ha M, Chen J, Zhang X, et al. Relationships of social support, healthpromoting lifestyles, glycemic control, and bone turnover among adults with type 2 diabetes. Jpn J Nurs Sci 2020;17:e12280.

40 Siegmann MJ, Athinarayanan SJ, Hallberg SJ, et al. Improvement in patient-reported sleep in type 2 diabetes and prediabetes participants receiving a continuous care intervention with nutritional ketosis. Sleep Med 2019;55:92-9. 